\title{
ANÁLISIS BIBLIOMÉTRICO MUNDIAL DE LA CATEGORÍA 'REMOTE SENSING’ DE LA WEB OF SCIENCE (1997-2012)
}

Global bibliometric analysis of the 'Remote Sensing' subject category from the Web of Science (1997-2012)

\section{JOSÉ IGNACIO ROJAS-SOLA ${ }^{1}$ ÁNGEL INOCENCIO AGUILERA-GARCÍA}

\author{
${ }^{1}$ University of Jaen \\ Department of Engineering Graphics, Design and Projects, Campus de las \\ Lagunillas \\ Jaén 23071 - Spain. \\ ${ }^{2}$ University of Jaen \\ Department of Computer Science, Campus de las Lagunillas \\ Jaén 23071 - Spain. \\ jirojas@ujaen.es ; angel@ujaen.es
}

\begin{abstract}
RESUMEN
El objetivo principal del trabajo ha sido analizar la evolución de la investigación en la categoría 'Remote Sensing', revisándose en la Web of Science, las revistas que se encuentran en dicha categoría, y analizándose bibliométricamente las publicaciones de dichas revistas para la serie temporal 1997-2012. Para ello, se han analizado para los diferentes países y centros de investigación, diversos indicadores bibliométricos como el número de documentos, y mostrándose por documento, la productividad, el número medio de citas, de autores, de centros de investigación, la colaboración nacional e internacional incluidas sus redes, el factor de impacto ponderado y relativo, así como el h-index. Asimismo, se ha analizado la difusión internacional de las investigaciones de los países a través de las revistas y la relación con el factor de impacto, para detectar las revistas de publicación de cada país. Por otro lado, se muestra cómo el inglés es el lenguaje de publicación por excelencia, consolidándose USA como el país más productivo aunque con un factor de impacto relativo bajo. También se muestra cómo la Chinese Academy of Sciences junto a la National Aeronautics and Space Administration (NASA) son las instituciones más productivas y el gran crecimiento de publicaciones de algunas universidades chinas.
\end{abstract}


Palabras clave: Bibliometría; Categoría 'Remote Sensing'; Producción Científica; Science Citation Index-Expanded; Journal Citation Reports.

\title{
RESUMO
}

O principal objetivo do presente estudo foi analisar a evolução da pesquisa em 'Sensoriamento Remoto', revisando na Web of Science, as revistas que estão nessa categoria, e analisando bibliometricamente as publicações dessas revistas para a série temporal 1997-2012. Para fazer isso, analisamos para diferentes países e centros de pesquisa, vários indicadores bibliométricos, como o número de documentos, e mostrando, por documento, a produtividade, o número médio de citações, de autores, de centros de pesquisa, a colaboração nacional e internacional, incluindo suas redes, o fator de impacto ponderado e relativo, bem como o índice h. Além disso, analisamos a divulgação internacional das pesquisas nos diversos países, através das revistas e a relação com o fator de impacto para detectar os periódicos publicados em cada país. Por outro lado, mostra-se como o Inglês é a linguagem de publicação por excelência, consolidando os USA como o mais produtivo, embora com um fator de impacto relativo baixo. Por outro lado se mostra como a Chinese Academy of Sciences junto com a National Aeronautics and Space Administration Nacional (NASA) são as instituições mais produtivas, e o grande crescimento de publicações de algumas universidades chinesas.

Palavras-chave: Bibliometria; Categoria 'Remote Sensing'; Produção Científica; Science Citation Index-Expanded; Journal Citation Reports.

\begin{abstract}
This study aimed at analyzing the evolution of the research into 'Remote Sensing' subject category; as a first approximation, we have revised in the Web of Science, the journals that are in that category. Secondly, we used bibliometric indicators to analyze publications for journals between 1997 and 2012. To do so, we analyzed for different countries and research centers, various bibliometric indicators such as the number of documents, showing, the productivity, the average number of citations, authors, research centers, national and international collaboration per document including their networks, the weighted and relative impact factor, as well as the hindex. Furthermore, we analyzed the international dissemination of research of countries through journals and the relationship with the impact factor to detect the published journals of each country. We have shown that English is the most common language of publication, and the USA is the most productive country, although it has a relatively low impact factor. We can remark that the Chinese Academy of Sciences and the National Aeronautics and Space Administration (NASA) are the most productive institutions, and the great number of publications of some Chinese universities.
\end{abstract}

Keywords: Bibliometric; 'Remote Sensing' subject category; Scientific Production; Science Citation Index-Expanded; Journal Citation Reports. 


\section{INTRODUCCIÓN}

El análisis bibliométrico es una herramienta especialmente útil a la hora de diseñar estrategias relacionadas con la producción científica, permitiendo esbozar líneas de actuación de política científica desde un punto de vista institucional que redunden en una mejora de la visibilidad de sus investigaciones.

En este artículo se analizan detalladamente las publicaciones científicas de las revistas de la categoría 'Remote Sensing' de los listados del Journal Citation Reports proporcionado por Thomson Reuters durante el período 1997-2012; es decir, el estudio abarca todo el período del que se dispone de información cuantitativa y cualitativa.

El objetivo principal de esta investigación inédita ha sido realizar una revisión, a través de la Web of Science (WoS), de los trabajos sobre 'Remote Sensing' realizados en países, universidades, instituciones o centros de investigación y publicados en revistas de dicha categoría, presentes en la base de datos en el período 1997-2012, justificado por el hecho de que se presentará un estudio de indicadores bibliométricos cuantitativos y cualitativos, lo que permitirá conocer mejor el estado actual de las investigaciones en esta área temática.

Trabajos de tipo institucional han sido realizados por el autor, así como por otros autores y han sido publicados en otras revistas internacionales (CANASGUERRERO et al., 2013, 2014; ROJAS-SOLA et al., 2008; ROJAS-SOLA y JORDA-ALBINANA, 2009a, 2009b, 2010, 2011; ROJAS-SOLA et al., 2009; ROJAS-SOLA y SAN-ANTONIO-GOMEZ, 2010a, 2010b, 2010c, 2010d, 2010e, 2010f).

El análisis cualitativo de las publicaciones científicas, se puede realizar de diversas formas: mediante el número de citas recibidas (WHITEHOUSE, 2001), a través del índice H (BALDOCK et al., 2009), del Eigenfactor (DAVIS, 2008), o mediante el Factor de Impacto (IF) publicado por Thomson Reuters (GARFIELD, 1994), entre otros. Dicho factor se refiere a una revista, y para un año determinado es el cociente entre las citas obtenidas en ese año, procedentes de los artículos publicados en los dos años anteriores, y el número de artículos publicados en los dos años anteriores, siendo todavía hoy día el sistema más ampliamente aceptado por la comunidad científica y administradores académicos, pese a las numerosas críticas que pueda tener (AMIN y MABE, 2003; GARFIELD, 1996, 2003). Sin embargo, lo que no se puede negar es el interés que despiertan este tipo de investigaciones bibliométricas, y además cuando no existen datos objetivos de la situación de dicha categoría a nivel mundial.

Asimismo, se ha realizado una revisión bibliográfica, y tan sólo se han encontrado dos artículos de revistas de la categoría que utilizan técnicas de análisis bibliométricos en sus estudios (SHORTIS, 2008; VAN DER MEER, 2012), pero no existe ningún estudio a nivel mundial donde se realice un análisis pormenorizado. Asimismo, hay un estudio de redes sociales desde la información espacial (DELAZARI y BRANDALIZE, 2012), que muestra la importancia de conocer esas redes de colaboración como se muestra en el presente artículo. 
Esta investigación muestra quiénes son los países, universidades, instituciones o centros de investigación más productivos y cuáles son las características cualitativas de sus publicaciones, para potenciar los puntos fuertes y corregir los débiles, pues sólo teniendo esta perspectiva será posible analizar la evolución de la producción científica en dicha área. Por lo tanto, se trata de una importante reflexión que puede ayudar en la toma de decisiones de carácter estratégico en el marco de política científico-tecnológica. Básicamente, estas decisiones consistirán tanto para un país, un centro de investigación o un investigador individual, en identificar las revistas más importantes de la categoría en función de su visibilidad internacional definida por su posición mantenida en las primeras posiciones de los listados del Journal Citation Reports durante los años, así como en el establecimiento de redes de colaboración con los centros de investigación más productivos y de mayor calidad científica basada en sus publicaciones. Por ello, es muy importante, caracterizar el área identificando dichos centros de investigación.

\section{MATERIAL Y MÉTODOS}

\subsection{Fuente de información para la extracción de la producción científica}

La base de datos escogida para el análisis de la producción científica en la categoría 'Remote Sensing' ha sido Science Citation Index-Expanded (SCI-E), cuyo proveedor es Thomson Reuters a través de su plataforma en línea del Web of Science (WoS).

\subsection{Proceso de extracción de la muestra de estudio}

Se ha realizado la extracción de todos los registros de todas las revistas de la categoría 'Remote Sensing' durante el período seleccionado. El proceso ha consistido en la descarga de todos los registros de la WoS, con los cuales se ha construido una base de datos utilizando el software Microsoft Access, y una vez construida se han realizado diferentes consultas en función de la información necesitada. Así pues, se han encontrado todas los tipologías documentales (article, proceedings paper, editorial material, review, correction, biographical-item, letter, news ítems, software review, bibliography, item about an individual, reprint, book review y database review), y posteriormente se ha aplicado un refinamiento por documento tipo (article or review, en adelante documentos) eliminándose registros de los que no se tenía constancia de país asignado ni de investigador. Además, se han revisado todos los registros debido a la falta de normalización en la denominación de los diferentes centros de investigación.

\subsection{Construcción de la base de datos específica para el análisis}

Tras la descarga de datos se ha construido una base de datos 'ad hoc' con toda la información integrada y de forma relacionada que permite operar de modo sencillo, flexible y rápido, con los distintos análisis de indicadores bibliométricos (MOED et al., 1995; VAN RAAN, 1999). Para ello, se ha utilizado la base de datos

Bol. Ciênc. Geod., sec. Artigos, Curitiba, v. 20, nº 4, p.855-878, out-dez, 2014. 
Microsoft Access para las cargas, modelado y tratamiento de información procedente de la base de datos SCI-E. Dicha base de datos adjudica un documento a cada uno de los países o centros de investigación, permitiendo un recuento múltiple, siempre y cuando aparezca en el campo Research Address de la base de datos, lo que se justifica ya que se trata de un estudio institucional, obteniéndose una serie de indicadores que permiten realizar tanto un análisis cuantitativo como cualitativo.

\subsection{Análisis cuantitativo}

Las variables contabilizadas y analizadas han sido:

Ndoc: Número de documentos adjudicado a cada país o centro de investigación. Un documento firmado por autores de diferentes filiaciones contabilizará por igual en cada país o centro de investigación.

Am: Número medio de autores por documento que corresponden a un país o centro de investigación.

$C m$ : Número medio de centros de investigación por documento.

CN: Número de documentos en colaboración nacional por país.

CI: Número de documentos en colaboración internacional por país.

CMC: Número de documentos de colaboración entre autores del mismo centro de investigación.

$\boldsymbol{C D C}$ : Número de documentos de colaboración entre autores de distintos centros de investigación.

\subsection{Análisis cualitativo}

Las variables analizadas han sido:

Citm: Número medio de citas por documento que corresponden a un país o centro de investigación.

h-index: Índice de Hirsch asignado a cada país o centro de investigación.

IFm \& IFr: Factor de Impacto medio por documento asignado a un país o centro de investigación. Para evitar los sesgos que se pueden producir, se ha normalizado el IF. El proceso seguido ha sido calcular el Impacto Total de un país o centro de investigación, y para ello, el criterio adoptado ha sido que a cada documento se le asigna el IF que tenga la revista en el año de publicación de dicho documento, y así sucesivamente para todos y cada uno de los documentos de cada país o centro de investigación, con lo que la suma será el IT del país o centro de investigación. Después se divide por el total de documentos de dicho país o centro de investigación, obteniéndose el Factor de Impacto medio (IFm).

Asimismo, se ha calculado el Factor de Impacto relativo (IFr) que se define como el IFm de cada país o centro de investigación, dividido por el IFm de la serie, con lo que se podrá conocer la posición relativa de cada uno de los países o centros de investigación en dicha serie. Aunque este procedimiento no está normalizado, conociendo que el IF es un valor propio de la revista y no del documento tipo, y dado que el estudio es nacional e institucional, esta metodología supone una primera aproximación del impacto de las publicaciones de cada una de ellas. 


\section{RESULTADOS Y DISCUSIÓN}

\subsection{Publicaciones en la categoría 'Remote Sensing'}

Una vez realizada la descarga, refinamiento y extracción de los datos se ha obtenido la producción científica clasificada por tipología documental. Así se han encontrado 27.686 trabajos de todos los tipos documentales como article (24.519), proceedings paper (1.506), editorial material (867), review (283), correction (206), biographical-item (158), letter (76), news ítem (33), software review (22), bibliography (2), item about an individual (8), reprint (4), book review (1) and database review (1), con datos consultados el 30 de octubre de 2013.

Asimismo, el número de documentos, ha crecido desde los 871 del año 1997 a los 3.036 del año 2012, lo que supone un crecimiento aproximado de un 348\%; el número de centros de investigación ha crecido desde los 1.393 en 1997 a los 7.076 en 2012 (508\%), y el número de autores ha pasado de los 2.079 en 1997 a los 12.057 en 2012 (580\%). También se ha podido observar cómo las citas, de documentos más antiguos, van disminuyendo a medida que la fecha de la publicación se aproxima al tiempo presente, así como que la colaboración internacional por países y centros de investigación presenta un suave crecimiento, mayor entre centros de investigación que a nivel global de países.

Además, dicho estudio ha permitido identificar las revistas y el número de documentos de cada una de ellas en la serie temporal estudiada (1997-2012) resultando que de las 31 revistas científicas que han existido en la categoría en dicha serie temporal, sólo 3 revistas aglutinan el 51,46\% del total de documentos: International Journal of Remote Sensing (20,80\%), IEEE Transactions on Geoscience and Remote Sensing (17,33\%) and Remote Sensing of Environment $(13,33 \%)$.

Asimismo se han analizado la totalidad de documentos por idioma de publicación, resultando que 24.475 están escritos en inglés, lo que supone el 98,68\% del total, evidenciando claramente, como era esperado, la necesidad de publicar en dicho idioma para obtener una buena difusión y visibilidad de los resultados (WAHEED, 2001). También se han publicado en otros idiomas como el Portugués (156), Alemán (51), Croata (45), Francés (40), Italiano (32), Español (2) y Rumano (1), lo que muestra efectivamente el valor residual de los otros idiomas.

Por otro lado, el factor de impacto medio por documento ha ido oscilando entre un valor de 1,24 para el año 1997 hasta uno de 2,29 para el año 2012, aunque presenta una leve tendencia alcista.

Asimismo, el número medio de autores por documento ha crecido siempre pasando de 2,39 en 1997 a 3,97 en 2012, y también el número medio de centros de investigación por documento, pasando de 1,60 en 1997 a 2,33 en 2012, aunque éste ha presentado oscilaciones en el período temporal estudiado. También el número medio de citas por documento alcanza un valor máximo en 1998 y en 2002, pero presenta una clara tendencia descendente. 


\subsection{Tendencias de investigación}

Las tendencias en investigación dentro de la categoría, se han obtenido tras el análisis de los principales keywords (author keywords y keywords plus). Así pues, se han analizado los 50 author keywords y 50 keywords plus más frecuentes, para la serie temporal y para cada uno de los 4 sub-períodos estudiados (1997-2000, 20012004, 2005-2008 y 2009-2012).

Dentro de los author keywords, destaca Remote Sensing con 1.670 apariciones y junto con Synthetic Aperture Radar (SAR) con 873 mantienen casi siempre las 2 primeras posiciones. Sin embargo, a diferencia de los keywords plus, las diez primeras posiciones no están tan agrupadas, oscilando sensiblemente en función del sub-período estudiado. Merece la pena señalar que hay determinados author keywords que han experimentado un crecimiento espectacular a medida que avanza el sub-período entre los cuales destacan Moderate Resolution Imaging Spectroradiometer (MODIS) con 655 apariciones que estaba en la posición 33 en el sub-período 1997-2000 y ha subido hasta la posición 3 en el último sub-período 2009-2012. Lo mismo ocurre con Light Detection and Ranging o Laser Imaging Detection and Ranging (LIDAR) con 329 apariciones que estaba en la posición 42 en el sub-período 1997-2000 y ha subido hasta la posición 4 en el último subperíodo 2009-2012, con Landsat con 298 apariciones que estaba en la posición 43 en el sub-período 1997-2000 y ha subido hasta la posición 5 en el último subperíodo 2009-2012, o con Land cover con 140 veces que estaba en la posición 44 y ha subido hasta la 15, aunque hay keywords que son actualidad debido al lanzamiento de un nuevo satélite, a la incorporación de nueva tecnología o la consolidación de una existente.

Dentro de los keywords plus, destacan tres que siempre han mantenido su posición en los 3 primeros puestos durante toda la serie temporal: Models con 2.355 apariciones, seguido por Images con 2.022, y Vegetation con 1.572, y otros como Classification con 1.540 y Algorithm con 1.405. Otros también importantes por el número de apariciones y que habitualmente ocupan las diez primeras posiciones son: Reflectance (767), Retrieval (674) y Accuracy (623). Por tanto, el topic más comúnmente usado ha sido en Model tanto en su expresión en singular como en plural con 2.355 apariciones.

Asimismo, existen topics que han subido notablemente como Validation con 533 apariciones que estaba en la posición 50 en el sub-período 1997-2000 y ha subido hasta la posición 6 en el último sub-período 2009-2012, o con MODIS con 468 apariciones que estaba en la posición 48 en el sub-período 1997-2000 y ha subido hasta la posición 11 en el último sub-período 2009-2012, y otros que experimentan un claro retroceso tanto en los author keywords como en los keywords como Scattering y Bidirectional reflectance.

\subsection{Actividad investigadora por país}

Por otro lado, se muestra un análisis por países para toda la serie temporal considerando los 50 más productivos con indicación del número de documentos, el 
porcentaje de documentos en colaboración nacional e internacional, el factor de impacto medio por documento, el número medio de citas por documento, el número medio de autores por documento, el número medio de centros de investigación por documento y el h-index (Tabla 1).

Tabla 1 - Indicadores bibliométricos por país (1997-2012).

\begin{tabular}{|c|c|c|c|c|c|c|c|c|c|c|c|}
\hline Country & Ndoc & $\begin{array}{l}\mathrm{CN} \\
(\%)\end{array}$ & $\mathrm{CN}$ & $\begin{array}{c}\text { CI } \\
(\%)\end{array}$ & CI & $\begin{array}{l}\text { IF } \\
\text { m }\end{array}$ & IFr & Citm & Am & $\mathrm{Cm}$ & $\begin{array}{c}\mathrm{h}- \\
\text { index }\end{array}$ \\
\hline USA & 9.780 & 56,6 & 5.539 & 43,3 & 4.234 & 1,55 & 0,85 & 19,85 & 3,85 & 3,79 & 201 \\
\hline China & 3.325 & 32,2 & 1.069 & 67,7 & 2.250 & 1,85 & 1,01 & 10,65 & 4,07 & 4,01 & 74 \\
\hline Canada & 2.079 & 45,3 & 941 & 54,7 & 1.137 & 1,74 & 0,95 & 14,53 & 3,67 & 3,61 & 86 \\
\hline Germany & 1.899 & 44,5 & 845 & 55,5 & 1.054 & 1,84 & 1,01 & 12,79 & 3,96 & 3,91 & 64 \\
\hline Italy & 1.796 & 54,0 & 970 & 45,9 & 825 & 1,69 & 0,92 & 16,65 & 4,08 & 3,97 & 76 \\
\hline France & 1.769 & 43,6 & 771 & 56,2 & 994 & 1,84 & 1,01 & 19,66 & 4,58 & 4,40 & 89 \\
\hline India & 1.426 & 71,8 & 1.024 & 28,2 & 402 & 1,37 & 0,75 & 5,44 & 3,55 & 3,51 & 41 \\
\hline England & 1.422 & 40,9 & 581 & 58,9 & 838 & 1,86 & 1,02 & 17,88 & 3,74 & 3,64 & 74 \\
\hline Australia & 1.131 & 41,5 & 469 & 58,5 & 662 & 1,81 & 0,99 & 12,74 & 3,79 & 3,67 & 58 \\
\hline Japan & 1.022 & 50,3 & 514 & 49,4 & 505 & 1,69 & 0,92 & 11,95 & 4,15 & 3,93 & 54 \\
\hline Netherlands & 1.012 & 34,0 & 344 & 65,9 & 667 & 2,04 & 1,12 & 17,75 & 4,31 & 4,14 & 66 \\
\hline Spain & 1.008 & 47,5 & 479 & 52,2 & 526 & 1,74 & 0,95 & 16,94 & 4,40 & 4,30 & 68 \\
\hline Brazil & 589 & 50,8 & 299 & 49,2 & 290 & 1,70 & 0,93 & 13,07 & 3,62 & 3,73 & 42 \\
\hline Taiwan & 482 & 34,0 & 164 & 65,8 & 317 & 1,91 & 1,04 & 10,94 & 3,71 & 3,63 & 38 \\
\hline Russia & 467 & 53,3 & 249 & 46,7 & 218 & 1,66 & 0,91 & 7,30 & 3,44 & 3,43 & 30 \\
\hline Finland & 449 & 53,2 & 239 & 46,5 & 209 & 1,76 & 0,96 & 18,20 & 4,59 & 4,38 & 55 \\
\hline Switzerland & 444 & 28,2 & 125 & 71,8 & 319 & 2,10 & 1,15 & 16,98 & 4,33 & 4,23 & 48 \\
\hline Greece & 348 & 61,5 & 214 & 38,5 & 134 & 1,51 & 0,83 & $9,, 03$ & 3,30 & 3,16 & 27 \\
\hline South Korea & 344 & 27,0 & 93 & 73,0 & 251 & 1,85 & 1,01 & 10,35 & 3,47 & 3,45 & 31 \\
\hline
\end{tabular}

Bol. Ciênc. Geod., sec. Artigos, Curitiba, v. 20, nº 4, p.855-878, out-dez, 2014. 
Rojas-Sola, J. I. ; Aguilera-García, A; I.

\begin{tabular}{|c|c|c|c|c|c|c|c|c|c|c|c|}
\hline Belgium & 316 & 44,6 & 141 & 55,4 & 175 & 1,87 & 1,02 & 15,42 & 4,27 & 4,17 & 40 \\
\hline Turkey & 310 & 65,8 & 204 & 34,2 & 106 & 1,43 & 0,78 & 7,40 & 2,72 & 2,71 & 25 \\
\hline Sweeden & 309 & 52,4 & 162 & 46,9 & 145 & 1,73 & 0,95 & 14,14 & 3,54 & 3,41 & 35 \\
\hline Austria & 299 & 28,1 & 84 & 71,9 & 215 & 2,14 & 1,17 & 17,75 & 4,25 & 4,07 & 39 \\
\hline Norwey & 264 & 42,0 & 111 & 58,0 & 153 & 1,88 & 1,03 & 17,58 & 4,02 & 4,01 & 40 \\
\hline Denmark & 236 & 34,3 & 81 & 65,7 & 155 & 1,99 & 1,09 & 19,15 & 4,11 & 3,82 & 39 \\
\hline Scotland & 215 & 35,8 & 77 & 64,2 & 138 & 1,88 & 1,03 & 13,74 & 3,92 & 3,66 & 29 \\
\hline Israel & 195 & 51,8 & 101 & 47,7 & 93 & 1,63 & 0,89 & 12,08 & 3,34 & 3,25 & 27 \\
\hline Portugal & 191 & 33,5 & 64 & 66,0 & 126 & 1,94 & 1,06 & 14,42 & 4,15 & 4,11 & 30 \\
\hline Iran & 186 & 55,4 & 103 & 44,6 & 83 & 1,53 & 0,84 & 4,90 & 3,19 & 3,14 & 15 \\
\hline Wales & 136 & 16,9 & 23 & 83,1 & 113 & 2,37 & 1,30 & 28,60 & 4,78 & $\begin{array}{c}4 ., 5 \\
7\end{array}$ & 41 \\
\hline South Africa & 131 & 32,1 & 42 & 67,9 & 89 & 2,03 & 1,11 & 10,31 & 4,15 & 3,01 & 24 \\
\hline Singapore & 130 & 23,1 & 30 & 76,9 & 100 & 2,05 & 1,12 & 11,45 & 3,70 & 2,61 & 21 \\
\hline $\begin{array}{c}\text { New } \\
\text { Zealand }\end{array}$ & 124 & 34,7 & 43 & 65,3 & 81 & 2,03 & 1,11 & 11,48 & 3,94 & 2,79 & 20 \\
\hline Malaysia & 101 & 22,8 & 23 & 77,2 & 78 & 2,08 & 1,14 & 8,20 & 3,46 & 2,17 & 17 \\
\hline Argentina & 97 & 34,0 & 33 & 66,0 & 64 & 2,06 & 1,13 & 11,69 & 4,58 & 3,22 & 24 \\
\hline Mexico & 90 & 45,6 & 41 & 54,4 & 49 & 1,79 & 0,98 & 10,48 & 4,41 & 3,76 & 18 \\
\hline Poland & 72 & 51,4 & 37 & 48,6 & 35 & 1,76 & 0,96 & 8,32 & 3,15 & 2,90 & 14 \\
\hline Ukraine & 68 & 42,6 & 29 & 57,4 & 39 & 1,82 & 0,99 & 3,47 & 4,01 & 2,57 & 9 \\
\hline Thailand & 66 & 25,8 & 17 & 74,2 & 49 & 2,17 & 1,19 & 6,20 & 3,62 & 2,23 & 14 \\
\hline $\begin{array}{c}\text { Czech } \\
\text { Republic }\end{array}$ & 65 & 21,5 & 14 & 78,5 & 51 & 2,32 & 1,27 & 9,45 & 4,08 & 2,94 & 16 \\
\hline Estonia & 61 & 39,3 & 24 & 60,7 & 37 & 1,97 & 1,08 & 17,70 & 3,69 & 2,73 & 21 \\
\hline Croatia & 50 & 92,0 & 46 & 8,0 & 4 & 1,09 & 0,60 & 0,52 & 2,22 & 2,33 & 4 \\
\hline Egypt & 50 & 50,0 & 25 & 50,0 & 25 & 1,63 & 0,89 & 4,42 & 3,14 & 2,50 & 10 \\
\hline
\end{tabular}

Bol. Ciênc. Geod., sec. Artigos, Curitiba, v. 20, nº 4, p.855-878, out-dez, 2014. 


\begin{tabular}{c|c|c|c|c|c|c|c|c|c|c|c}
\hline Slovenia & 50 & 42,0 & 21 & 58,0 & 29 & 1,65 & 0,90 & 16,46 & 2,90 & 1,87 & 14 \\
\hline Hungary & 48 & 29,2 & 14 & 70,8 & 34 & 2,33 & 1,27 & 10,42 & 4,63 & 3,57 & 16 \\
\hline Indonesia & 48 & 16,7 & 8 & 83,3 & 40 & 2,41 & 1,32 & 7,33 & 5,10 & 3,57 & 9 \\
\hline Kenya & 43 & 18,6 & 8 & 81,4 & 35 & 2,15 & 1,18 & 11,84 & 4,09 & 3,03 & 13 \\
\hline Chile & 41 & 19,5 & 8 & 80,5 & 33 & 2,47 & 1,35 & 7,93 & 3,88 & 2,87 & 12 \\
\hline Sri Lanka & 40 & 7,5 & 3 & 92,5 & 37 & 3,03 & 1,66 & 27,33 & 5,75 & 3,75 & 17 \\
\hline Iceland & 37 & 13,5 & 5 & 86,5 & 32 & 2,32 & 1,27 & 30,92 & 4,11 & 2,86 & 15 \\
\hline
\end{tabular}

Respecto a los indicadores cuantitativos, de dicha tabla se desprende cómo los cinco países más productivos son USA (9.780), China (3.325), Canada (2.079), Germany (1.899) e Italy (1.796), suponiendo el $27,49 \%$, el $9,35 \%$, el $5,84 \%$, el $5,34 \%$ y el $5,05 \%$ respectivamente del total de documentos, sumando un 53,07\% de total de países encontrados (135).

Sin embargo, a pesar de ser los más productivos no coinciden, salvo USA, con los que más destacan en número medio de citas por documento, destacando de forma sobresaliente Iceland $(30,92)$, seguida por Wales $(28,60)$, Sri Lanka $(27,33)$, USA $(19,85)$, France $(19,66)$ y Denmark $(19,15)$.

Asimismo, la colaboración nacional e internacional en el ámbito de los países ha puesto de manifiesto, que países como Croatia (92,0\%), India (71,8\%), Turkey $(65,8 \%)$, Greece $(61,5 \%)$ y USA $(56,6 \%)$ son los países con mayor colaboración entre Centros de Investigación del mismo país, y que los países con mayor colaboración internacional son Sri Lanka (92,5\%), Iceland (86,5\%), Indonesia $(83,3 \%)$, Wales $(83,1 \%)$ y Chile $(80,5 \%)$.

También se ha podido analizar el número medio de autores por documento destacando Sri Lanka (5,75), Indonesia $(5,10)$, Wales $(4,78)$, Hungary $(4,63)$ y Finland $(4,59)$, así como el número medio de centros de investigación por documento, destacando igualmente Finland $(4,38)$, Spain $(4,30)$, Switzerland $(4,23)$, Belgium $(4,17)$ y Netherlands $(4,14)$.

Respecto a los indicadores cualitativos, se ha analizado el IFm de cada país, para lo que se ha contado con el valor del IF de cada revista en cada de la serie temporal, resultando que destacan países como Sri Lanka $(3,03)$, Chile $(2,47)$, Indonesia $(2,41)$, Wales $(2,37)$ y Hungary $(2.33)$, y por el contrario otros son los 5 últimos como Iran $(1,53)$, Greece $(1,51)$, Turkey $(1,43)$, India $(1,37)$ y Croatia $(1,09)$.

Finalmente la Tabla 1, muestra un valor h-index para USA (201) muy superior al de resto de países como France (89), Canada (86), Italy (76), China (74) y England (74), lo que es sin duda, una evidencia de la calidad de las publicaciones de 
dicho país al presentar un gran número de artículos que son citados. Sin embargo, hay que matizar este resultado pues el número de documentos en USA (9.780) es muy superior al resto, con lo que estableciendo una comparación de dicho indicador frente al número de documentos tipo, resulta que USA y China ocuparían los últimos lugares en este indicador, destacando países como Wales, Denmark, Portugal, Norway, Israel y Scotland, si bien es cierto que es mucho más difícil mantener la misma ratio de estos países cuando el número de documentos es muy elevado.

\subsection{Actividad investigadora por Centro de Investigación}

En primer lugar, es preciso subrayar que el proceso de búsqueda por centros de investigación en la base de datos construida ad hoc, se realiza con la cadena de términos exacta que aparece en los registros de la base de datos SCI-E, asumiendo el error que se pueda producir al no estar normalizado el proceso y por tanto, es probable que un mismo centro de investigación figure con expresiones distintas. Teniendo en cuenta esta observación, se ha realizado una búsqueda lo más completa posible de los 50 centros de investigación más productivos del mundo con sus diferentes expresiones.

Respecto a los indicadores cuantitativos, del análisis institucional (Tabla 2), hay que destacar que hay dos centros de investigación que destacan por el número de documentos como la Chinese Academy of Sciences (1.236) y National Aeronautics and Space Administration (NASA) (1.159), seguidas de lejos por la University of California (691), United States Department of Agriculture (USDA) (619), California Institute of Technology (CALTECH) (615) y University of Maryland (580).

Sin embargo, a pesar de ser los más productivos, no todos ellos destacan en número medio de citas por documento, destacando 5 centros norteamericanos como University of Maryland con un valor de 41,27, seguido por Boston University $(40,79)$, University of Wisconsin $(39,77)$, Oregon State University $(37,70)$ y NASA $(34,59)$.

Tabla 2 - Indicadores bibliométricos por Centros de Investigación (19972012).

\begin{tabular}{c|c|c|c|c|c|c|c|c|c|c|c|c}
\hline $\begin{array}{c}\text { Research } \\
\text { Center }\end{array}$ & Country & Ndoc & $\begin{array}{c}\text { CMC } \\
(\%)\end{array}$ & CMC & $\begin{array}{c}\text { CDC } \\
(\%)\end{array}$ & CDC & IFm & IFr & Citm & Am & Cm & $\begin{array}{c}\text { h- } \\
\text { index }\end{array}$ \\
\hline $\begin{array}{c}\text { Chinese } \\
\text { Academy of } \\
\text { Sciences } \\
\text { (CAS) }\end{array}$ & China & 1.236 & 43,4 & 533 & 56,4 & 701 & 1,85 & 1,09 & 10,60 & 4,49 & 2,92 & 50 \\
\hline $\begin{array}{c}\text { National } \\
\text { Aeronautics } \\
\text { and Space } \\
\text { Administration } \\
\text { (NASA) }\end{array}$ & USA & 1.159 & 42,0 & 488 & 57,9 & 670 & 1,96 & 1,16 & 34,59 & 5,27 & 3,42 & 90 \\
\hline $\begin{array}{c}\text { University of } \\
\text { California }\end{array}$ & USA & 691 & 54,3 & 388 & 45,7 & 303 & 1,70 & 1,01 & 26,03 & 4,36 & 2,90 & 66 \\
\hline
\end{tabular}


Análisis bibliométrico mundial de la categoría 'Remote Sensing'...

\begin{tabular}{|c|c|c|c|c|c|c|c|c|c|c|c|c|}
\hline $\begin{array}{l}\text { United States } \\
\text { Department of } \\
\text { Agriculture } \\
\text { (USDA) }\end{array}$ & USA & 619 & 45,4 & 284 & 54,3 & 333 & 1,89 & 1,12 & 26,33 & 5,04 & 3,42 & 63 \\
\hline $\begin{array}{l}\text { California } \\
\text { Institute of } \\
\text { Technology } \\
\text { (CALTECH) }\end{array}$ & USA & 615 & 53,8 & 328 & 46,2 & 287 & 1,70 & 1,01 & 25,14 & 5,06 & 3,48 & 60 \\
\hline $\begin{array}{c}\text { University of } \\
\text { Maryland }\end{array}$ & USA & 580 & 36,9 & 214 & 63,1 & 366 & 2,03 & 1,20 & 41,27 & 4,98 & 3,37 & 75 \\
\hline $\begin{array}{c}\text { National } \\
\text { Oceanic and } \\
\text { Atmospheric } \\
\text { Administration } \\
\text { (NOAA) }\end{array}$ & USA & 430 & 45,8 & 198 & 54,2 & 232 & 1,96 & 1,16 & 24,80 & 5,10 & 3,39 & 49 \\
\hline $\begin{array}{c}\text { Germany's } \\
\text { Aerospace } \\
\text { Research } \\
\text { Center and } \\
\text { Space Agency } \\
\text { (DLR) }\end{array}$ & Germany & 407 & 82,6 & 331 & 17,4 & 76 & 1,22 & 0,72 & 14,19 & 4,13 & 2,51 & 36 \\
\hline $\begin{array}{l}\text { Indian Space } \\
\text { Research } \\
\text { Organization } \\
\text { (ISRO) }\end{array}$ & India & 404 & 81,4 & 329 & 18,6 & 75 & 1,21 & 0,72 & 4,59 & 4,00 & 2,60 & 21 \\
\hline $\begin{array}{l}\text { Ohio State } \\
\text { University }\end{array}$ & USA & 376 & 60,4 & 226 & 39,6 & 150 & 1,62 & 0,95 & 11,52 & 4,10 & 2,60 & 32 \\
\hline $\begin{array}{c}\text { Beijing } \\
\text { Normal } \\
\text { University }\end{array}$ & China & 370 & 36,2 & 133 & 63,8 & 237 & 2,12 & 1,25 & 13,19 & 4,68 & 3,14 & 34 \\
\hline $\begin{array}{c}\text { National } \\
\text { Research } \\
\text { Council of } \\
\text { Italy (CNR) } \\
\end{array}$ & Italy & 359 & 84,1 & 292 & 15,6 & 66 & 1,24 & 0,73 & 16,03 & 4,42 & 2,51 & 42 \\
\hline $\begin{array}{c}\text { Commission } \\
\text { European } \\
\text { Communities } \\
\end{array}$ & Europe & 353 & 68,8 & 248 & 31,2 & 105 & 1,40 & 0,83 & 22,85 & 4,03 & 2,50 & 45 \\
\hline $\begin{array}{c}\text { Wuhan } \\
\text { University }\end{array}$ & China & 340 & 47,6 & 160 & 52,4 & 180 & 1,72 & 1,02 & 7,60 & 4,08 & 2,70 & 25 \\
\hline $\begin{array}{c}\text { United States } \\
\text { Geological } \\
\text { Survey } \\
\text { (USGS) } \\
\end{array}$ & USA & 333 & 56,0 & 188 & 44,0 & 145 & 1,72 & 1,02 & 30,40 & 4,64 & 2,98 & 52 \\
\hline $\begin{array}{l}\text { Natural } \\
\text { Resources } \\
\text { Canada }\end{array}$ & Canada & 317 & 44,9 & 142 & 55,1 & 175 & 1,83 & 1,08 & 18,03 & 4,22 & 2,71 & 37 \\
\hline $\begin{array}{c}\text { China } \\
\text { Meteorogical } \\
\text { Administration } \\
\text { (CMA) }\end{array}$ & China & 305 & 43,6 & 131 & 56,1 & 173 & 1,96 & 1,16 & 17,93 & 4,69 & 2,78 & 31 \\
\hline $\begin{array}{c}\text { Indian Institute } \\
\text { of Technology } \\
\text { (IIT) }\end{array}$ & India & 300 & 71,7 & 222 & 28,3 & 78 & 1,28 & 0,76 & 8,38 & 3,50 & 2,36 & 31 \\
\hline $\begin{array}{l}\text { United States } \\
\text { Navy (USN) }\end{array}$ & USA & 294 & 57,1 & 167 & 42,9 & 127 & 1,60 & 0,95 & 14,76 & 4,47 & 2,84 & 33 \\
\hline $\begin{array}{c}\text { Boston } \\
\text { University }\end{array}$ & USA & 293 & 36,9 & 109 & 63,1 & 184 & 2,38 & 1,41 & 40,79 & 5,71 & 4,08 & 55 \\
\hline $\begin{array}{l}\text { University of } \\
\text { Twente }\end{array}$ & $\begin{array}{c}\text { Netherlan } \\
\text { ds }\end{array}$ & 271 & 73,8 & 200 & 26,2 & 71 & 1,38 & 0,82 & 13,56 & 4,22 & 2,05 & 31 \\
\hline
\end{tabular}

Bol. Ciênc. Geod., sec. Artigos, Curitiba, v. 20, nº 4, p.855-878, out-dez, 2014. 
Rojas-Sola, J. I. ; Aguilera-García, A; I.

867

\begin{tabular}{|c|c|c|c|c|c|c|c|c|c|c|c|c|}
\hline $\begin{array}{l}\text { University of } \\
\text { Colorado }\end{array}$ & USA & 270 & 46,7 & 127 & 53,3 & 143 & 1,78 & 1,05 & 24,21 & 4,94 & 3,25 & 40 \\
\hline $\begin{array}{c}\text { University of } \\
\text { Calgary }\end{array}$ & Canada & 261 & 70,9 & 183 & 29,1 & 78 & 1,39 & 0,82 & 10,92 & 3,08 & 2,17 & 27 \\
\hline $\begin{array}{l}\text { National } \\
\text { Institute for } \\
\text { Space } \\
\text { Research } \\
\text { (INPE) }\end{array}$ & Brazil & 240 & 81,3 & 196 & 18,8 & 44 & 1,23 & 0,73 & 17,70 & 4,08 & 2,70 & 31 \\
\hline $\begin{array}{c}\text { University of } \\
\text { Wisconsin }\end{array}$ & USA & 225 & 37,8 & 86 & 62,2 & 139 & 1,96 & 1,16 & 39,77 & 4,88 & 3,22 & 45 \\
\hline $\begin{array}{c}\text { European } \\
\text { Space Agency } \\
\text { (ESA) } \\
\end{array}$ & Europe & 218 & 75,8 & 128 & 23,7 & 90 & 1,56 & 0,92 & 17,48 & 5,94 & 2,98 & 33 \\
\hline $\begin{array}{c}\text { National } \\
\text { Research } \\
\text { Council of } \\
\text { France } \\
\text { (CNRS) } \\
\end{array}$ & France & 211 & 67,6 & 109 & 31,4 & 101 & 1,67 & 0,99 & 22,61 & 5,54 & 2,65 & 40 \\
\hline $\begin{array}{c}\text { University of } \\
\text { Valencia }\end{array}$ & Spain & 210 & 20,6 & 144 & 79,4 & 64 & 1,40 & 0,83 & 21,70 & 4,93 & 3,21 & 38 \\
\hline $\begin{array}{c}\text { Oregon State } \\
\text { University }\end{array}$ & USA & 199 & 74,1 & 41 & 25,9 & 158 & 2,57 & 1,52 & 37,70 & 5,88 & 4,26 & 47 \\
\hline $\begin{array}{l}\text { Commonwealt } \\
\text { h Scientific } \\
\text { and Industrial } \\
\text { Research } \\
\text { Organization } \\
\text { (CSIRO) }\end{array}$ & Australia & 193 & 89,5 & 142 & 10,5 & 51 & 1,45 & 0,86 & 17,18 & 4,39 & 2,68 & 32 \\
\hline $\begin{array}{c}\text { Delft } \\
\text { University of } \\
\text { Technology } \\
\end{array}$ & $\begin{array}{l}\text { Netherlan } \\
\text { ds }\end{array}$ & 190 & 42,0 & 169 & 58,0 & 21 & 1,13 & 0,67 & 14,14 & 2,97 & 1,91 & 27 \\
\hline $\begin{array}{l}\text { Russian } \\
\text { Academy of } \\
\text { Sciences }\end{array}$ & Russia & 188 & 85,6 & 160 & 14,4 & 28 & 1,20 & 0,71 & 7,07 & 3,54 & 2,56 & 20 \\
\hline $\begin{array}{c}\text { Colorado State } \\
\text { University }\end{array}$ & USA & 188 & 42,4 & 80 & 57,6 & 108 & 1,91 & 1,13 & 18,22 & 4,64 & 3,14 & 31 \\
\hline $\begin{array}{c}\text { University of } \\
\text { Arizona }\end{array}$ & USA & 184 & 60,9 & 113 & 39,1 & 71 & 1,62 & 0,96 & 29,41 & 4,20 & 3,08 & 39 \\
\hline $\begin{array}{c}\text { Canada Centre } \\
\text { for Remote } \\
\text { Sensing } \\
(\text { CCRS })\end{array}$ & Canada & 184 & 29,0 & 77 & 71,0 & 107 & 1,78 & 1,05 & 18,91 & 4,09 & 2,44 & 33 \\
\hline $\begin{array}{c}\text { Nanjing } \\
\text { University }\end{array}$ & China & 183 & 37,5 & 53 & 62,5 & 130 & 2,09 & 1,24 & 6,08 & 4,49 & 2,84 & 18 \\
\hline $\begin{array}{c}\text { National } \\
\text { Center for } \\
\text { Spatial Studies } \\
\text { (CNES) }\end{array}$ & France & 181 & 46,2 & 99 & 53,2 & 82 & 1,70 & 1,01 & 19,88 & 5,83 & 2,70 & 35 \\
\hline $\begin{array}{c}\text { Hong Kong } \\
\text { Polytechnic } \\
\text { University }\end{array}$ & China & 176 & 56,8 & 65 & 43,2 & 111 & 1,91 & 1,13 & 7,39 & 3,86 & 2,77 & 20 \\
\hline $\begin{array}{l}\text { University of } \\
\text { Washington }\end{array}$ & USA & 173 & 47,0 & 80 & 53.0 & 92 & 1,87 & 1,11 & 18,90 & 4,75 & 3,40 & 29 \\
\hline $\begin{array}{l}\text { National } \\
\text { Institute of } \\
\text { Agronomic } \\
\text { Research } \\
\text { (INRA) } \\
\end{array}$ & France & 173 & 46,6 & 75 & 51,6 & 97 & 1,92 & 1,14 & 24,78 & 5,77 & 2,91 & 36 \\
\hline
\end{tabular}

Bol. Ciênc. Geod., sec. Artigos, Curitiba, v. 20, nº 4, p.855-878, out-dez, 2014. 
Análisis bibliométrico mundial de la categoría 'Remote Sensing'...

\begin{tabular}{c|c|c|c|c|c|c|c|c|c|c|c|c}
\hline $\begin{array}{c}\text { University of } \\
\text { Texas }\end{array}$ & USA & 169 & 39,4 & 96 & 60,0 & 73 & 1,61 & 0,95 & 12,04 & 4,10 & 2,60 & 23 \\
\hline $\begin{array}{c}\text { University of } \\
\text { Waterloo }\end{array}$ & Canada & 168 & 35,1 & 81 & 64,9 & 87 & 1,73 & 1,02 & 12,83 & 3,79 & 2,55 & 24 \\
\hline $\begin{array}{c}\text { Xidian } \\
\text { University }\end{array}$ & China & 161 & 61,4 & 75 & 37,9 & 83 & 1,77 & 1,05 & 7,22 & 4,29 & 3,24 & 17 \\
\hline $\begin{array}{c}\text { George Mason } \\
\text { University }\end{array}$ & USA & 160 & 60,7 & 63 & 39,3 & 96 & 1,81 & 1,07 & 8,33 & 4,18 & 2,96 & 19 \\
\hline $\begin{array}{c}\text { University of } \\
\text { British } \\
\text { Columbia }\end{array}$ & Canada & 154 & 84,0 & 55 & 16,0 & 99 & 1,95 & 1,15 & 16,10 & 4,31 & 3,05 & 29 \\
\hline $\begin{array}{c}\text { University of } \\
\text { Michigan }\end{array}$ & USA & 153 & 73,8 & 94 & 25,5 & 58 & 1,51 & 0,89 & 16,64 & 3,31 & 2,27 & 28 \\
\hline $\begin{array}{c}\text { National } \\
\text { Central } \\
\text { University }\end{array}$ & Taiwan & 150 & 42,3 & 90 & 57,7 & 60 & 1,60 & 0,95 & 10,75 & 4,03 & 2,60 & 22 \\
\hline $\begin{array}{c}\text { University of } \\
\text { Nottingham }\end{array}$ & England & 150 & 78,2 & 126 & 21,1 & 24 & 1,26 & 0,75 & 29,20 & 3,29 & 1,99 & 33 \\
\hline $\begin{array}{c}\text { Politechnic } \\
\text { University of } \\
\text { Catalonia }\end{array}$ & Spain & 149 & 51,0 & 108 & 49,0 & 40 & 1,35 & 0,80 & 12,36 & 5,65 & 3,96 & 25 \\
\hline $\begin{array}{c}\text { State Oceanic } \\
\text { Administration }\end{array}$ & China & 149 & 63,2 & 61 & 36,8 & 88 & 1,83 & 1,08 & 11,50 & 4,34 & 2,51 & 22 \\
\hline
\end{tabular}

Asimismo se ha analizado, el número de documentos en los que hubo la colaboración entre investigadores del mismo centro y de distinto centro de investigación, pues no ha sido posible la distinción a nivel internacional ya que en muchos registros faltaba la información relativa al país.

Así pues, la colaboración entre investigadores del mismo centro de investigación ha subrayado cómo destacan Instituciones como Commonwealth Scientific and Industrial Research Organization (CSIRO) (89,50\%), Russian Academy of Sciences (85,60\%), National Research Council of Italy (CNR) (84,10\%), University of British Columbia (84\%) y Germany's Aerospace Research Center and Space Agency (DLR) (82,60\%), y respecto a la colaboración entre distintos centros de investigación, destacan Oregon State University (79,4\%), Nanjing University (71\%), University of British Columbia (64,9\%), Beijing Normal University (63,8\%), University of Maryland y Boston University $(63,1 \%)$.

También, se ha podido analizar el número medio de autores por documento destacando European Space Agency (ESA) (5,94), Oregon State University $(5,88)$, National Center for Spatial Studies of France (CNES) (5,83), National Institute of Agronomic Research of France (INRA) (5,77) y Boston University (5,71), así como el número medio de centros de investigación por documento, destacando igualmente Oregon State University (4,26), Boston University (4,08), Polytechnic University of Catalonia (3,96), CALTECH $(3,48)$, USDA y NASA $(3,42)$.

Respecto a los indicadores cualitativos, se ha analizado el IFm de cada centro de investigación, destacando Oregon State University (2,57), Boston University (2,38), Beijing Normal University (2,12), Nanjing University $(2,09)$ y University of Maryland (2,03), y subrayando que hay centros de investigación que no llegan al valor de la unidad para el IFr de la serie temporal, y concretamente los 5 últimos del 
ranking son CNR (0,73), National Institute for Space Research (INPE) (0,73), Indian Space Research Organization (ISRO) $(0,72)$, DLR $(0,72)$ y Russian Academy of Sciences $(0,71)$. Este último indicador (IFr) puede ser menor de la unidad ya que sería el cociente entre el IFm de cada centro de investigación y el IFm medio de la serie. Así pues, si el IFm del centro es menor que el IFm medio de la serie, el IFr será menor de la unidad, lo que permite establecer una comparación sin sesgo y conocer la posición relativa de cada centro respecto a la serie.

Finalmente la Tabla 2, muestra un valor h-index para la NASA (90) superior al de resto de centros de investigación como University of Maryland (75), Universiy of California (66), USDA (63) y CALTECH (60), lo que evidencia la calidad de las publicaciones de dichos centros de investigación. Sin embargo, hay que matizar este resultado pues el número de documentos varía significativamente entre centros de investigación, con lo que estableciendo una comparación de dicho indicador frente al número de documentos, resulta que las instituciones mejor clasificadas serían Oregon State University, University of Nottingham, University of Arizona, National Institute of Agronomic Research (INRA) y University of Wisconsin.

Así pues, se observa cómo la Chinese Academy of Sciences ha subido desde la posición 7 hasta la 1 en el último sub-período (2009-2012), siendo en este período cuando realiza numerosas aportaciones a la categoría situándola en la posición 1 en el cómputo global durante el período estudiado (1997-2012), mientras que la NASA ha permanecido siempre en la primera posición hasta este último sub-período descendiendo a la posición 2, pero permaneciendo en dicha posición en el cómputo global. Por otro lado, la University of California siempre se mantiene entre las posiciones 3 y 4 . Asimismo, destaca USDA que pasa de la posición 6 a la 3, y de forma sobresaliente Beijing Normal University, Wuhan University, DLR, China Meteorological Administration (CMA), University of Twente, Nanjing University y Xidian University, apreciando el gran empuje de diversos centros de investigación chinos. Asimismo, destaca de forma negativa, la importante disminución de documentos de la Russian Academy of Sciences que ha pasado de la posición 5 en el período 1997-2000 hasta la 50 en el último período analizado (2009-2012).

\subsection{Redes de colaboración nacional e internacional entre países y centros de investigación}

Tras analizar la colaboración nacional e internacional en las tablas 1 y 2 , se ha podido realizar una búsqueda en la base de datos para concretar la relación internacional existente entre países, estableciendo los porcentajes en número de documentos colaborados, y de igual forma, la relación nacional e internacional entre centros de investigación, y por tanto, definiendo las redes de colaboración a nivel nacional e internacional.

La figura 1 muestra la red de colaboración internacional entre los 10 países más productivos y la figura 2 muestra la red de colaboración nacional e internacional entre los 10 centros de investigación con mayor producción científica. 
Figura 1 - Red y porcentajes de colaboración internacional entre cada país y sus 3 máximos colaboradores de entre los 10 más productivos a nivel mundial. Para USA se muestran todas las colaboraciones.

PERCENTAGE OF INTERNATIONAL COLLABORATION BETWEEN COUNTRIES (1997-2012)

Figura 2 - Red y porcentajes de colaboración internacional entre cada centro de investigación y sus 3 máximos colaboradores de entre los 10 más productivos a nivel mundial. Para NASA y CAS se muestran todas las colaboraciones. 




Del análisis de la colaboración internacional entre países (Figura 1), lo primero a señalar es que efectivamente, USA es el primer país de colaboración internacional con los más productivos, destacando China con un 49,38\% de su producción científica, India con un $35,03 \%$, Canada con un $31,26 \%$, Australia con un 26,63\% y Japan con un $26,48 \%$. Asimismo, y en sentido inverso, la colaboración de USA con China representa un $25,26 \%$ de su producción científica, con Canada un 8,78\%, con France un 6,28\%, con Germany un 5,18\% y con Australia un 4,56\%.

Entre el resto de los 10 países más productivos, la relación más fuerte de colaboración se centra entre Japan y China representando un 20,55\% de la producción científica de Japan, seguida por la relación de Australia con China $(12,61 \%)$, China con Canada (11,16\%), Italy con France $(10,49 \%)$ y England con Germany $(9,25 \%)$.

El estudio a nivel de país se ha realizado con todos los países, resaltando el elevado porcentaje de colaboración entre USA y los 'Four Asian Tigers' (South Korea $(54,73 \%)$, Taiwan $(42,43 \%)$ y en menor medida, Singapore $(14,71 \%)$ y Hong Kong $(5,55 \%))$.

También destaca de forma sobresaliente la colaboración de algunos países que aun no siendo los más productivos, sí presentan altos porcentajes de colaboración, 
como Israel con USA $(44,78 \%)$, Brazil con USA $(41,84 \%)$ o Turkey con USA $(33,33 \%)$ de su producción científica.

Por otro lado, del análisis de colaboración nacional e internacional entre los 10 centros de investigación más productivos (Figura 2), se puede decir que los dos principales centros de investigación entre los cuales se concentran un gran número de colaboraciones son la Chinese Academy of Sciences y la NASA. Por un lado, los centros de investigación que más colaboran, expresado en porcentaje sobre el número de documentos con la Chinese Academy of Sciences son University of Maryland (5,83\%), University of California (5,25\%) y Ohio State University $(3,91 \%)$, y con la NASA son la University of Maryland (12,83\%), National Oceanic and Atmospheric Administration (NOAA) (7,47\%) y USDA (7,03\%).

Entre el resto de los 10 centros de investigación más productivos, la colaboración es escasa o nula, centrándose la más fuerte entre Ohio State University y CALTECH (3,75\%), NOAA y University of Maryland (3,58\%), y CALTECH y University of California (2,23\%).

Asimismo, se aprecia cómo existen 2 centros de investigación sin ninguna relación con el resto de entre los 10 más productivos, como son $D L R$ y ISRO, siendo para cada uno de ellos, centros de investigación de su ámbito nacional sus 3 principales colaboradores.

Del análisis realizado, también se observa que la mayoría de centros de investigación estatales de USA como NOAA, USDA, United States Geological Survey (USGS) y United States Navy (USN), tienen a la NASA como su principal colaborador.

También destaca de forma sobresaliente la colaboración de algunos centros de investigación que aun no siendo los más productivos, sí presentan altos porcentajes de colaboración, como por ejemplo, CCRS y Natural Resources Canada (14,21\%), University of Nottingham y University of Southampton (12,82\%), University of British Columbia y Natural Resources Canada (12,75\%), Indian Institute of Technology (ITT) e ISRO (12,57\%), Oregon State University y NASA (10,43\%) o University of Michigan y CALTECH $(9,71 \%)$.

Finalmente, otro hecho destacable es que existen un gran número de centros de investigación chinos que encuentran a la Chinese Academy of Sciences su principal colaborador como Hong Kong Polytechnic University (16,35\%), Nanjing University (16,05\%), Beijing Normal University (12,81\%), Wuhan University (12,46\%), Xidian University (12,25\%), CMA (9,89\%), National Central University (9,02\%) y State Oceanic Administration (8,71\%).

\subsection{Internacionalización y difusión de las revistas}

Del estudio realizado, sólo 9 revistas de la categoría en la serie temporal estudiada presentan publicaciones de cada uno de los 30 países más productivos: International Journal of Remote Sensing, IEEE Transactions on Geoscience and Remote Sensing, Remote Sensing of Environment, Radio Science, Photogrammetric Engineering and Remote Sensing, IEEE Geoscience and Remote Sensing Letters, 
Canadian Journal of Remote Sensing, ISPRS Journal of Photogrammetry and Remote Sensing y Remote Sensing. Además todas ellas figuran en las 10 primeras posiciones de las revistas con mayor número de documentos, y 6 de ellas siempre han presentado factor de impacto durante todos los años de la serie temporal (19972012). En la Tabla 3 se muestran los factores de impacto de dichas revistas para todos los años de la serie temporal.

Tabla 3 - Factores de Impacto de las revistas (1997-2012).

\begin{tabular}{|c|c|c|c|c|c|c|c|c|c|c|c|c|c|c|c|c|}
\hline Revista & 1997 & 1998 & 1999 & 2000 & 2001 & 2002 & 2003 & 2004 & 2005 & 2006 & 2007 & 2008 & 2009 & 2010 & 2011 & 2012 \\
\hline $\begin{array}{c}\text { Canadian } \\
\text { Journal of } \\
\text { Remote Sensing } \\
\end{array}$ & & & & & & & 0,593 & 1,284 & 0,862 & 0,752 & 0,658 & 1,315 & 0,724 & 0,962 & 0,560 & 0,986 \\
\hline $\begin{array}{c}\text { IEEE Geoscience } \\
\text { and Remote } \\
\text { Sensing Letters }\end{array}$ & & & & & & & & & & & 1,140 & 1,832 & 1,379 & 1,431 & 1,560 & 1,823 \\
\hline $\begin{array}{c}\text { IEEE } \\
\text { Transactions on } \\
\text { Geoscience and } \\
\text { Remote Sensing } \\
\end{array}$ & 1,419 & 1,251 & 1,732 & 1,485 & 1,605 & 1,603 & 1,867 & 1,467 & 1,627 & 1,752 & 2,344 & 3,157 & 2,234 & 2,485 & 2,895 & 3,467 \\
\hline $\begin{array}{c}\text { International } \\
\text { Journal of } \\
\text { Remote Sensing }\end{array}$ & 0,771 & 0,829 & 0,979 & 0.827 & 0,827 & 1,154 & 0,990 & 1,128 & 0,925 & 0,980 & 0,987 & 1,041 & 1,089 & 1,188 & 1,117 & 1,138 \\
\hline $\begin{array}{c}\text { ISPRS Journal of } \\
\text { Photogrammetry } \\
\text { and Remote } \\
\text { Sensing }\end{array}$ & 0,283 & 0,132 & 0,492 & 0,692 & 0,963 & 0,389 & 0,472 & 1,317 & 1,674 & 1,381 & 1,116 & 2,293 & 2,308 & 2,184 & 2,885 & 3.313 \\
\hline $\begin{array}{l}\text { Photogrammetric } \\
\text { Engineering and } \\
\text { Remote Sensing }\end{array}$ & 1,103 & 0,781 & 1,006 & 0,783 & 0,841 & 1,176 & 0,857 & 1,574 & 1,000 & 1,284 & 1,103 & 1,846 & 1,110 & 0,931 & 1,048 & 1,802 \\
\hline Radio Science & 1,059 & 0,944 & 0,860 & 0,922 & 1,139 & 0,796 & 0,832 & 1,007 & 0,951 & 1,084 & 0,972 & 1,092 & 1,012 & 0,950 & 1,075 & 1,000 \\
\hline Remote Sensing & & & & & & & & & & & & & & & & 2,101 \\
\hline $\begin{array}{l}\text { Remote Sensing } \\
\text { of Environment }\end{array}$ & 2,198 & 1,410 & 1,868 & 1,888 & 1,697 & 1,992 & 2,641 & 3,185 & 2,833 & 3,064 & 3,013 & 3,943 & 3,612 & 3,954 & 4,574 & 5,103 \\
\hline
\end{tabular}

USA es el país con mayor número de contribuciones en dichas revistas salvo en la Canadian Journal of Remote Sensing, donde únicamente Canada presenta más publicaciones (328 frente a 208).

Por países, se puede apreciar cómo USA publica principalmente en tres revistas: IEEE Transactions on Geoscience and Remote Sensing (20,91\%), Remote Sensing of Environment (18,07\%) y International Journal of Remote Sensing $(17,90 \%)$, agrupando un total del $56,88 \%$ de toda su producción científica; China publica fundamentalmente en tres revistas: International Journal of Remote Sensing (26,30\%), IEEE Transactions on Geoscience and Remote Sensing (15,55\%) y IEEE Geoscience and Remote Sensing Letters (10,94\%), agrupando un total del 52,79\% de su producción científica; Canada publica fundamentalmente en tres revistas: International Journal of Remote Sensing (19,54\%), Remote Sensing of Environment $(16,46 \%)$ y Canadian Journal of Remote Sensing (15,78\%), agrupando un total del $51,78 \%$ de su producción científica; Germany publica fundamentalmente en tres revistas: IEEE Transactions on Geoscience and Remote Sensing (15,75\%), 
International Journal of Remote Sensing $(14,74 \%)$ y Journal of Geodesy $(12,85 \%)$, agrupando un total del $43,34 \%$ de su producción científica; Italy publica fundamentalmente en tres revistas: IEEE Transactions on Geoscience and Remote Sensing (29,08\%), International Journal of Remote Sensing (18,22\%) y Remote Sensing of Environment (11,42\%), agrupando un total del 58,72\% de su producción científica; France publica fundamentalmente en tres revistas: IEEE Transactions on Geoscience and Remote Sensing (23,17\%), Remote Sensing of Environment $(21,70 \%)$ y International Journal of Remote Sensing (19,72\%) agrupando un total del $64,59 \%$ de su producción científica; India publica fundamentalmente en dos revistas: International Journal of Remote Sensing $(35,06 \%)$ y Journal of the Indian Society of Remote Sensing (28,67\%), agrupando un total del $63,73 \%$ de su producción científica; England publica fundamentalmente en tres revistas: International Journal of Remote Sensing $(29,46 \%)$, Remote Sensing of Environment $(15,15 \%)$ y IEEE Transactions on Geoscience and Remote Sensing (13,53\%), agrupando un total del $58,14 \%$ de su producción científica; Australia publica fundamentalmente en tres revistas: International Journal of Remote Sensing (16,71\%), Remote Sensing of Environment (13,35\%) y IEEE Transactions on Geoscience and Remote Sensing (10,88\%), agrupando un total del 40,94\% de su producción científica, y Japan publica fundamentalmente en tres revistas: International Journal of Remote Sensing (25.91\%), IEEE Transactions on Geoscience and Remote Sensing (21,59\%) y Radio Science (12,95\%) agrupando un total del $60,45 \%$ de su producción científica.

\section{CONCLUSIONES}

En este artículo se aplica el análisis bibliométrico a la categoría del Web of Science 'Remote Sensing' para definir la evolución de la investigación en esta área temática y realizar un análisis mundial de los países más productivos e igualmente un estudio institucional de las principales universidades, instituciones y centros de investigación, lo que sin duda ofrece una visión global de la actividad investigadora en los últimos años y puede ser muy útil a la hora de planificar estrategias a nivel nacional e institucional de cara a tener una mayor visibilidad.

Se ha identificado la evolución de los topics de investigación destacando que las tendencias vienen marcadas por el uso de los keywords plus más frecuentes, entre los cuales destacan Model, Classification, Vegetation manteniéndose siempre entre las 3 primeras posiciones. Sin embargo, existen otros que están experimentando un notable crecimiento en los que se encuentran Retrieval, Accuracy, Validation, System y Moderate Resolution Imaging Spectroradiometer (MODIS). Por el contrario, existen otros que están experimentando un claro retroceso en su utilización como Scattering, Bidirectional Reflectance or Inversion.

Analizando el comportamiento de los author keywords, es necesario señalar que los que siempre aparecen en las 2 primeras posiciones son Remote Sensing and Synthetic Aperture Radar (SAR), aunque también hay varios que experimentan un rápido crecimiento como Laser Imaging Detection and Ranging (LiDAR), Landsat, 
Change Detection or Land Cover, o un claro retroceso como Scattering, Image processing or Modeling.

Por otro lado, se ha determinado la evolución de las publicaciones en un contexto geográfico e institucional, demostrándose que en 30 países se concentra el $94 \%$ de las publicaciones mundiales de un total de 135 países, mayoritariamente en inglés $(98 \%)$.

Igualmente se ha comprobado cómo el aumento del factor de impacto medio presenta una leve tendencia lineal de crecimiento debido también al aumento del número de publicaciones, aunque a diferencia del número de documentos esta tendencia no siempre ha sido creciente presentando oscilaciones en la serie temporal.

Por otro lado, la colaboración internacional se ha incrementado notablemente en lo relativo al número de autores por documento (de 2,39 a 3,97) y al número de centros de investigación por documento (de 1,60 a 2,33), aun presentando oscilaciones en ambos casos. Esto viene a subrayar la complejidad a la hora de establecer las redes de colaboración.

Respecto a la actividad investigadora por países, es preciso señalar que 30 países aglutinan el $94,02 \%$ de las publicaciones, destacando países como USA (9.780), China (3.325), Canada (2.079), Germany (1.899) e Italy (1.796), suponiendo el $27,49 \%$, el $9,35 \%$, el $5,84 \%$, el $5,34 \%$ y el $5,05 \%$ respectivamente del total de documentos tipo, sumando un 53,07\% de total de países encontrados (135). Se observa cómo los países del G8 (USA, Canada, Germany, Italy, France, England, Japan y Russia) figuran entre los 15 países más productivos, incluyendo además a países emergentes como China, India, Australia, Netherlands, Spain, Brazil and Taiwan, no destacando, sin embargo los 'Four Asian Tigers' (Hong Kong, Singapore, South Korea y Taiwan).

Por tanto, se puede apreciar cómo USA es el país con mayor número de publicaciones contando con 21 centros de investigación de entre los 30 más productivos, lo que supone un dominio absoluto, entre los que destacan de forma sobresaliente, NASA, University of California, USDA, CALTECH, University of Maryland y NOOA. También destaca USA como el país que presenta el h-index más alto (201) (201 documentos con al menos 201 citas), debido también a que presenta alrededor de 10 revistas con factor de impacto mayor de la unidad, aunque sin embargo, no ocurre lo mismo con el factor de impacto relativo que es menor de la unidad, pues al aumentar el número de artículos se publican en revistas con un impacto menor o sin impacto, lo que hace que disminuya el impacto medio de las publicaciones de USA.

Asimismo, es de destacar el gran crecimiento de las publicaciones de la Chinese Academy of Sciences sobre todo en el cuarto sub-período (2009-2012) que la han convertido en el primer centro de investigación mundial por número de publicaciones, y se constata además la tendencia creciente de diversas universidades chinas como Beijing Normal University, Wuhan University, Nanjing University, 
Hong Kong Polytechnic University, Xidian University, o la organización CMA, todas con un factor de impacto relativo superior a la unidad.

Por último, teniendo en cuenta los principales indicadores bibliométricos utilizados (IFm, Citm y h-index), si tuviéramos que destacar los mejores centros de investigación analizados que superan los valores medios de la serie de todos esos indicadores, serían estos 14: National Aeronautics and Space Administration (NASA), University of California, United States Department of Agriculture (USDA), California Institute of Technology (CALTECH), University of Maryland, National Oceanic and Atmospheric Administration (NOAA), United States Geological Survey (USGS), Natural Resources Canada, Boston University, University of Colorado, University of Wisconsin, National Research Council of France (CNRS), Oregon State University y National Institute of Agronomic Research of France (INRA).

Por último, se han detectado las principales revistas de publicación por país, así como las redes de colaboración entre países y entre centros de investigación, lo que puede ayudar a un investigador a posicionarse de cara a una máxima visibilidad de sus publicaciones.

\section{REFERENCIAS BIBLIOGRÁFICAS}

AMIN, M.; MABE, M.A. Impact factors: use and abuse. In: Medicina-Buenos Aires, 63, 347-354, 2003.

BALDOCK, C.; MA. R.M.S.; ORTON, C.G. The h index is the best measure of a scientist's research productivity. In: Medical Physics, 36, p. 1043-1045, 2009.

CANAS-GUERRERO, I.; MAZARRÓN, F.R.; POU-MERINA, A.; CALLEJAPERUCHO, C.; DIAZ-RUBIO, G. Bibliometric analysis of research activity in the field "Agronomy" category from the Web of Science, 1997-2011. In: European Journal of Agronomy, 50, p. 19-28, 2013.

CANAS-GUERRERO, I., MAZARRON, F.R., CALLEJA-PERUCHO, C, \& POUMERINA, A. (2014). Bibliometric analysis in the international context of the 'Construction \& Building Techonology' category from the Web of Science data base. In: Construction and Building Materials, 53, p. 13-25, $2013 \mathrm{c}$.

DAVIS, P.M. Eigenfactor: Does the principle of repeated improvement result in better estimates than raw citation counts?. In: Journal of the American Society for Information Science and Technology, 59, p. 2186-2188, 2008.

DELAZARI, L.S.; BRANDALIZE, M.C.B. An analysis of social networks from spatial information. In: Boletim de Ciencias Geodesicas, 18, 185-202, 2012.

GARFIELD, E. The impact factor. In: Current Content, 20, p. 3-7, 1994.

GARFIELD, E. Fortnightly review: how can impact factors be improved?. In: British Medical Journal, 313, p. 411-413, 1996.

GARFIELD, E. Measuring and assessing science beyond SCI. In: Current Science, 85, p. 425-425, 2003.

MOED, H.F.; BRUIN, H.F.; VAN LEEUWEN, T.N. New bibliometric tools for the assessment of national research performance: Database description, overview of indicators and firs application. In: Scientometrics, 33, p. 381-422, 1995. 
ROJAS-SOLA, J. I.; NAVARRETE CORTES, J.; FERNANDEZ LOPEZ, J.A.; CHAICHIO MORENO, J.A. Scientific production in the graphic expression in engineering area at the Spanish universities: An approach to ISI database. In: Revista Española de Documentacion Cientifica, 31, p. 190-204, 2008.

ROJAS-SOLA, J.I.; JORDA-ALBINANA, B. Scientific production in the graphic expression in engineering area at the Spanish universities: An approach to ISI Database. In: Interciencia, 34, p. 689-695, 2009a.

ROJAS SOLA, J.I.; JORDA-ALBINANA, B. (2009b). Bibliometric analysis of Spanish scientific publications in the subject 'Materials Science, Ceramics' in JCR (SCI) database (1997-2008). In: Boletin de la Sociedad Espanola de Ceramica y Vidrio, 48, p. 255-260, 2009 b.

ROJAS-SOLA, J.I.; JORDA-ALBINANA, B.; CRIADO-HERRERO, E. Bibliometric analysis of Latin American, Spanish and Portuguese scientific publications in the subject 'Materials Science, Ceramics' in JCR (SCI) database (1997-2008). In: Boletin de la Sociedad Espanola de Ceramica y Vidrio, 48, p. 297-310, 2009.

ROJAS-SOLA, J.I.; JORDA-ALBINANA, B. Bybliometric analysis of Venezuelan scientific publications in the 'Ecology' subject category in Web of Science (WoS) database (1997-2008). In: Interciencia, 35, p. 619-623, 2010.

ROJAS-SOLA, J.I.; SAN-ANTONIO-GÓMEZ, C. Bibliometric analysis of Uruguayan scientific publications in the 'Engineering, Chemical' in the Web of Science category (1997-2008). In: Ingeniería Química, 38, p. 33-37, 2010a.

ROJAS-SOLA, J.I.; SAN-ANTONIO-GÓMEZ, C. Bibliometric analysis of Spanish scientific publications in the subject 'Construction \& Building Technology' in Web of Science database (1997-2008). In: Materiales de Construcción, 60, p. 143-149, $2010 \mathrm{~b}$.

ROJAS-SOLA, J.I., \& SAN-ANTONIO-GÓMEZ, C. Bibliometric analysis of Colombian scientific publications in 'Engineering, Multidisciplinary' subject category in Web of Science database (1997-2009). In: Dyna-Colombia, 77, p. 9-17, 2010c.

ROJAS-SOLA, J.I., \& SAN-ANTONIO-GÓMEZ, C. (2010d). Bybliometric analysis of Argentinean scientific publications in the 'Agriculture, Multidisciplinary' subject category in Web of Science database (1997-2009). In: Revista de la Facultad de Ciencias Agrarias, 42, p. 71-83, $2010 \mathrm{~d}$.

ROJAS-SOLA, J.I.; SAN-ANTONIO-GÓMEZ, C. Bibliometric analysis of Mexican scientific publications in the category 'Engineering, Chemical' from the Web of Science database (1997-2008). In: Revista Mexicana de Ingenieria Quimica, 9, p. 231-240, 2010e.

ROJAS-SOLA, J.I.; SAN-ANTONIO-GÓMEZ, C. Bibliometric analysis of Spanish scientific publications in the subject 'Metallurgy \& Metallurgical Engineering' in Web of Science database (1997-2008). In: Revista de Metalurgia, 46, p. 555-562, $2010 f$. 
ROJAS-SOLA, J.I.; JORDA-ALBINANA, B. (2011). Bibliometric analysis of Mexican scientific production in hydraulic engineering based on journals in the Science Citation Index-Expanded database (1997-2008). In: Tecnologia y Ciencias del Agua, 2, p. 195-213, 2011.

SHORTIS, M.R. Standards quality and impact factor of an international journal of photogrammetry. In: Photogrammetric Record, 23, p. 457-459, 2008.

VAN DER MEER, F. Remote-sensing image analysis and geostatistics. In: International Journal of Remote Sensing, 33, p. 5644-5676, 2012.

VAN RAAN, A.F.J. Advanced bibliometric methods for the evaluation of Universities. In: Scientometrics, 45, p. 417-423, 1999.

WAHEED, A.A. Scientists turn to journals in English. In: Scientific World, I, p. 239-242, 2001.

WHITEHOUSE, G.H. Citation rates and impact factors: should they matter?. In: British Journal of Radiology, 74, p. 1-3, 2001.

(Recebido em abril de 2014. Aceito em agosto de 2014). 